\title{
Design of Nanostructured Lipid Carriers Ubiquinone-10 for Transdermal Treatment by Esti Hendradi
}

Submission date: 28-Oct-2018 08:10PM (UTC+0800)

Submission ID: 1028115537

File name: tured_Lipid_Carriers_Ubiquinone-10_for_Transdermal_Treatment.pdf (192.42K) Word count: 3672

Character count: 19956 
Available online on www.ijddt.com

International Journal of Drug Delivery Technology 2018; 8(3);116-120

Research Article

ISSN: 09754415

\title{
Design of Nanostructured Lipid Carriers Ubiquinone-10 for Transdermal Treatment
}

\author{
M Fatchur Rochman ${ }^{1,2}$, Isnaeni $^{1}$, Esti Hendradi ${ }^{1}$ \\ 'Department of Pharmaceutics, Faculty of Pharmacy, Airlangga University, Indonesia \\ ${ }^{2}$ Departmen of Pharmaceutics, Faculty of Pharmacy, Wahid Hasyim University, Indonesia
}

Received: $9^{\text {th }}$ Apr, 18; Revised: $25^{\text {th }}$ May, 18, Accepted: $17^{\text {th }}$ Jul, 18; Available Online: $25^{\text {th }}$ Sep, 2018

\begin{abstract}
Ubiquinone-10 is a cellular endogenous antioxidant that resides in the epidermal layer. Ubiquinone-10 can increase the production of basal membrane components, fibroblast proliferation and protect cells from oxidative damage, so with increasing age, the amount of Ubiquinone-10 body will decrease. Bioavailability and permeability of Ubiquinone-10 are very low, thus affecting the potential efficacy and absorption in Ubiquinone-10. The preparation of Ubiquinone-10 formulation needs to be done to obtain products that have good bioavailability. Nanostructured Lipid Carrier (NLCs) is a modification of the SLN system, consisting of a certain amount of lipid matrix and liquid lipids. Mixtures of solid lipids and liquid lipids can provide space for drug trapping. The system will remain in solid form by controlling the concentration of the liquid lipids to be added into the formula so that the drug release process can be controlled.
\end{abstract}

Keywords: Nanostructured Lipid Carriers, Ubiquinone-10, Transdermal Treatment.

\section{INTRODUCTION}

Ubiquinone-10 is an antioxidant that protects the body from damage caused by harmful molecules known as free radicals. Ubiquinone-10 can neutralize free radicals and help prevent damage caused by free radicals, such as cell membrane damage, DNA damage, and cell death. The antioxidant properties of Ubiquinone-10 come from the carrier function of energy. As an energy carrier, the molecules of Ubiquinone-10 will continuously pass through the redox cycle. When receiving electrons, it will be reduced and when giving electrons, it becomes oxidized. In reduced form, the Ubiquinone-10 molecule holds a somewhat loose electron, so that this Ubiquinone10 molecule will be quite easy to take one or both of the electrons, therefore acting as an antioxidant. Ubiquinone10 inhibits lipid peroxidation by preventing the production of peroxyl lipid radicals ${ }^{7,10}$.

Ubiquinone-10 is a derivative of a lipid-soluble quinone with an isoprenoid side chain. The homolog of Ubiquinone contains 1-12 isoprene units. The dominant form of ubiquinone in humans is ubiquinone-10 (contains 10 isoprene units). In liver cells, $40-50 \%$ of the total cellular ubiquinone is located in the mitochondria, $25-30 \%$ in the core, $15-20 \%$ in the endoplasmatic reticulum, and only 5 $10 \%$ in the cytosol. In vitro tests, ubiquinol (reduced form of ubiquinone) has antioxidant activity 2-3 times more potent. Ubiquinol/ubiquinone acts as a redox carrier in the respiratory chain, participating in the transfer of protons across the inner mitochondrial membrane. Ubiquinol can react with ROS and prevent direct damage to biomolecules and initiation of lipid peroxidation. Ubiquinone cannot prevent autocatalytic free radical reactions by donating a phenolic hydrogen atom, ubiquinone binding singlet oxygen and inhibiting lipid peroxidation in the membrane model $^{5}$.

Nanostructured Lipid Carrier (Nlc)

NLC is the development of SLN (Solid Lipid Nanoparticle) aimed at overcoming the shortcomings of SLN. SLN is identical to an oil-in-water emulsion, but the oil phase used is a solid lipid. The use of solid lipid causes the drug loading on this system is not good, as well as the occurrence of leaks or discharge of medicines from the system. In storage, the solid lipid will form a crystal structure so that the space occupied by the drug material becomes reduced. This is what causes the release of drugs from the SLN system. NLC formed from a mixture of solid and liquid lipids capable of reducing the formation of crystals in lipid and can increase drug absorption, NLC has a particle size distribution up to $500 \mu \mathrm{m}$. NLC is the latest generation of SLN. NLC consists of a certain amount of lipid matrix and liquid lipids. The NLC remains in solid form by controlling the liquid lipid content added to the formulation, so that the controlled release of the drug to NLC can be achieved ${ }^{17}$.

Another component added to the NLC system is the surfactant. The addition of surfactants in the NLC serves to stabilize the nanoparticles formed on the system and prevent the incorporation of particles during storage ${ }^{16}$. The components used in NLC formulations are included in GRAS (Generally Regarded as Safe) ${ }^{15}$. Solid lipids that can be used on NLC systems include; stearic acid, glyceryl stearate, cetyl palmitate, and tristearin ${ }^{15,18,19}$. While liquid lipids that can be used include oleic acid, ethyl oleate, and miglyol (medium-chain triglycerides) ${ }^{15,19}$. With the

"Author for Correspondence: estihendradi@yahoo.com 
increase of liquid lipid, it will decrease the measurement an particle. Due to the increase of solid lipids, the tendency of particles to aggregate also increases. An increase in the number of liquid lipids can result in better drug trapping. This may have an effect on the formation of crystals, so the regularity of the crystal lattice is disrupted and increases the space for drug molecules which ultimately increases the efficiency of trapping. The amount of addition of the surfactant used also affects the particle size and stability of the formula. Increased surfactant concentration can decrease particle size. The number and type of surfactant optimum are able to envelop the surface of the particles ${ }^{19}$. Type Of $N L C$

NLC Type 1

NLC type I can be called imperfect imperfect model (Imperfect type), because the structural difference between the solid and liquid lipids leads to imperfections of the formation of crystals to form a larger space for drug molecules to occupy. This model was obtained at the time of solid lipid mixing with small amounts of lipid liquid NLC Type II

NLC type II was defined as an amorphous model as it was created when mixing certain lipids that could not crystallize after mixing. The lipid used is a solid lipid with an amorphous form. In the absence of crystallization, the tendency of the drug to get out of the system can be reduced.

NLC Type III

NLC type III referred to as multiple models. This model has been developed to increase drug charge capacity. Multiple types is a type of NLC capable of loading drugs in solid lipids, while the increased solubility of drug substances is done by adding the number of liquid lipids. In general, the drug will be more soluble in liquid lipids than in solid lipids. So in the process of making the amount of liquid lipid will be higher. During the production process, there will be a cooling process at room temperature which causes the lipid matrix particles to change from liquid to solid. The difference in mixing between the two lipids leads to a separation that will form the oil nano-compartments inside the solid lipid particles. Advantages of $N L C$

NLC has the advantage of being used for controlled drug release and targeted drugs, improving pharmaceutical stability, increasing drug loading ${ }^{21}$. Some of the advantages of NLC are:

Occlusive effects

Occlusive effects are influenced by particle size, lipid concentration, and the type of lipid used. Very small particles of lipid particles form high crystals and melting points of lipids are low resulting in increased occlusivity properties ${ }^{4}$. The surface area of The particles in NLCs in contact with the stratum corneum can be increased and the lipids used can make the NLC become adhesive to the skin thereby increasing the penetration of the active ingredient through the stratum corneum and providing an occlusive effect on the skin ${ }^{13}$

Controlled drug release
The use of solid lipids in the NLC system causes the mobility of the drug substances in the lipids to be lower resulting in the release of drug substances gradually.

Improve drug stability

Increase in chemical stability due to the small contact of the drug with oxygen, light, moisture, ambient temperature and contamination due to drug ingredients trapped in the NLC system ${ }^{6}$. NLC is able to prevent the expulsion of medicinal materials from the system because it inhibits the formation of the lipid crystals so that the stability during storage can be increased.

Have low toxicity

The composite material of the NLC system consists of solid lipids and liquid lipids that have non-irritating properties, biodegradable and safe / Generally Recognized As Safe (GRAS) so that preparations with NLC systems have low toxicity ${ }^{14}$.

Increase drug trapping

High carrier capacity so that many drug molecules can be incorporated into matrix particles ${ }^{3}$

Having a low viscosity

NLC system has a low viscosity so that the molecular mobility of the active ingredient is increased so that there is no inhibitory barrier ${ }^{23}$.

Transport System Of Drug

The drug transport is a mass transport process with one random movement occurs by the influence of concentration differences. Movement of molecules through a barrier with a particular mechanism is a diffusion process. The process of transport occurs through a variety of mechanisms, including molecular permeation through various mechanisms, including molecular permeation through a non porous barrier where the molecule of the compound must be dissolved beforehand into the barrier before it can penetrate, whereas in the transport through the pore, the transport speed is determined by the measured molecular size and pore diameter. The drug transport mechanism of the dosage forms occurs in two stages. The first stage, drug release from the carrier, stage second a penetrate through the barrier. The main transport process occurs in passive diffusion, ie the transport process that occurs due to the difference in concentration gradient ${ }^{20}$.

Penetration Work

On percutaneous penetration occurs the transfer of active ingredients through the skin from the external environment to the blood circulation. Percutaneous absorption can be defined as a combination of molecular penetration through the skin, followed by resorption of the blood or lymphatic system of the papillary dermis and underlying layer. The penetration stage is a passive diffusion through each structure of the stratum corneum, dermis, aneksa ${ }^{2}$. The skin has several layers, among others, stratum corneum is a percutaneous absorption rate inhibitor. Large and hydrophilic molecular transport will be difficult to penetrate the lipid layer of the stratum corneum because it contains $40 \%$ lipid, $40 \%$ protein, and only $20 \%$ water. The transport of lipophilic drug molecules is facilitated by dissolution to the intercellular liquid present in the stratum corneum. The absorption of a hydrophilic drug may occur through the pore or opening of the follicle and sebaceous 
glands, but this occurs only about $1 \%$ of the total surface of the skin so that only a small amount of the drug is absorbed $^{11}$. Percutaneous absorption of the drug is important in transdermal drug delivery because the drug should be sufficiently dabbed to prolong the duration of action and increase the rate of the drug and to maintain systemic uniformity and therapeutic effects during the period of use. In general, drug molecules penetrate the stratum corneum to the deepest layer of the dermal layer to the systemic that occurs in a relatively short and easy time $^{11}$. The rate of drug penetration passes through the stratum corneum following the Fick Law diffusion. The drug transport rate is not only influenced by its solubility but also the oil and water partition coefficient, the concentration in the dosage and the exposed surface area ofthe skin ${ }^{11}$.

Drug Penetration Route

Commonly used methods for the drug to penetrate the stratum corneum via transepidermal and route through the pores. Transepidermal routes can be divided into transcellular and intercellular routes. On the transcellular route, a drug that penetrates into the skin directly passes through the lipid structure of the stratum corneum and cytoplasm of keratin. This route is the shortest route but the medicinal material undergoes a hydrophilic and lipophilic structure. The most frequent route of medication to penetrate the skin is the intercellular route so that the material that will penetrate the stratum corneum by passing through the corneocytes. Appendix of skin (glands and hair follicles) is only $0.1 \%$ of the total surface of the skin, the role of the route through the pore is considered not too large ${ }^{22}$.

Factors Affecting Skin Penetration

Factors that influence percutaneous penetration are physiology, physical chemistry ingredients, medicines, and factors of carrier substances.

Skin physiology

Skin aged Increasingly aging,

skin permeability will decrease as blood flow decreases so that drug delivery at target sites decreases and water loss from the epidermis ${ }^{8}$.

Skin condition Difficult skin due to irritation,

dryness, allergic reaction, abrasion, inflammation can injure protective cells so penetration through the skin will increase $^{8}$

Anatomy of skin

The Scientology resides like skin thickness, the skin will cause a difference in penetration. This is because the penetration depends on the anatomy and thickness of the membrane layer ${ }^{8}$.

Hydration of skin

Hydration can affect the skin tissue physically and will change the diffusion coefficient of the material

Blood flow

While the flow of blood increases then the drug ingredient to the systemic will increase. If there is a narrowing of blood vessels that lead to the dermis then the skin penetration rate will decrease ${ }^{8}$.

Skin Temperatures
The speed of drug penetration through the skin can change when the body has increased temperature. The temperature will increase in the occlusive state. In the occlusive state, water on the skin cannot evaporate and cause hydration so that the flow of blood in the dermal layer will increase ${ }^{8}$.

Physical properties of the active ingredients.

\section{Partition coefficient}

The partition coefficient is the ratio between the drug concentration in the stratum corneum and the drug levels in the carrier. If the partition coefficient is large, then the drug will be more soluble in the carrier. If the partition coefficient is small, the drug will be more soluble in the stratum corneum than in the drug carrier so that the drug content in the large stratum corneum. The partition coefficient affects the transport speed ${ }^{9}$.

The drug's cooling

The solution of the drug in the water determines the absorbed concentration. The more drug substances in the dissolved state the greater the drug's ability to penetrate the membrane?

\section{The diffusion coefficient}

The diffusion coefficients show the ability of the drug penetrate into the stratum corneum. Usually, the drug diffusion coefficients in the stratum corneum are very low due to the very slow movement of drug molecules ${ }^{1}$ ). If the skin condition in sickness or drug diffusion in the carrier is very slow, the percutaneous penetration rate is determined by drug release from the carrier ${ }^{1}$. Part shape and size The small particle size and spherical shape will cause the interparticle structure to become tightly causing an increase in the occlusivity of retained water in the stratum corneum which causes hydration and swelling so that the drug substances easily enter ${ }^{1}$.

\section{The carrier effect}

Carrier may also affect the penetration of the drug into the skin as most of the material in one formulation is a carrier. The interaction between drug and carrier material can affect penetration because the strong affinity between the drug and the carrier causes the drug substance to be removed from the base so that the amount of medicinal ingredient in the stratum corneum decreases. There are several factors that come from the carriers that come out have an effect on the penetration:

The nature of lipophilicity

Carrier can affect the physical condition and permeability of the skin against the drug. The carrier composition is expected to improve the speed and amount of the drug diffusion. The lipophilic carrier will block the water so that water can not get out and retained in the stratum corneum causing skin hydration. Hydration of the stratum corneum can increase penetration of the drug into the. The drug mixed with the carrier must be able to blend with the skin surface in sufficient concentration so that penetration can be maximized.

Viscosity

Absorption of the drug will increase if the carrier can easily spread to the skin surface so that penetration will increase. This can happen because when used the drug can spread to a wider surface resulting in increased absorption of the skin. 


\section{Enhancer}

Increased penetration of topical medication can be achieved by the addition of several compounds capable of increasing the absorption in the skin. One of the enhancer mechanisms is by making the interaction of enhancers with polar head groups from lipids. Interaction of lipid-lipid head groups and sequences of lipids will be disrupted thus facilitating the diffusion of hydrophilic drugs. The increased flow of free water molecules between bilayers, leading to enlargement of the cross section for polar drug diffusion. Simple hydration can be used in structura modifications that result in drug penetration changes. Water is one of the most effective and safest penetration enhancers. By hydration of the stratum corneum, penetration of most drugs can be increased. Usually in the stratum corneum moisture content of $5-10 \%$. Water content may increase by up to $50 \%$ in occlusion conditions (eg by using impermeable foil or by occlusive vehicle applications). The lipid head group disorder by polar enhancer substances may also affect the hydrophobic parts of the lipids and cause rearrangements in the bilayer region. It also explains the increased penetration of lipophilic drugs by using a lipid head group affects the penetration of hydrophilic enhancer substances. Another possibility is the lipophilic penetration enhancement interaction with the hydrocarbon chains of the lipid bilayer. Penetration of lipophilic drugs is facilitated in this way with a skeletal disorder resulting from increased fluidization by hydrocarbon chains. This change also affects the order of the polar head groups, which explains the increased penetration of hydrophilic drugs by using lipophilic enhancer substances ${ }^{22}$.

\section{CONCLUSIONS}

NLCs are expected to be an alternative treatment aimed at treatment for a long time and have targeted targets, then the drug can be used for the treatment of transdermal that takes a long time. It is hoped that the NLC can become a new, periodically transdermal treatment of transdermal treatment. So the treatment will be more efficient with good effectiveness, but the side effects will be less.

\section{REFERENCE}

1. Barry, B.W.. 2002. Drug Delivery Routes In Skin: A Novel Approach. Advanced Drug Delivery Reviews. 54 Suppl. 1.

2. Berard, F., Marty, J., P., Nicolas, J., F., 2003. Allergen Penetration Through The Skin. Eur J of Dermatol 13 (4)

3. Couvreur, P., Kante, B., Lenaerts V., Scaiteur, V., Roland, M., Speiser, P., 2002. Tissue Distribution of antitumor Drugs Associated with Polyalkylcyanoacrylate Nanoparticles. Inter Journal of Pharm. 69.

4. Dubey, A., Prabhu, P., Kamath, J.V. 2012. Nanostructured Lipid Carriers: A Novel Topical Drug Delivery System. International Journal of Pharmaceutical Technology Research. Vol.4. 2.
5. Elsner, P., Maibach, H., I., 2005. Cosmeceuticals and Active Cosmetics Drugs Versus Cosmetics, $2^{\text {nd }} \mathrm{Ed}$ Jungerman Associates, Inc. Phoenix, Arizona.

6. Hommoss, A., 2008. Nanostructured Lipid Carriers (NLC) In Dermal and Personal Care Formulations. Inaugural-Dissertation. Berlin. The Department of Biology, Chemistry, and Pharmacy of the Freie Universitat Berlin

7. Kapoor, A.K. 2013. Coenzyme Q10 - A Novel Molecule. JACM; 14(1).

8. Konda, Sales, M., D., Susan, R., Cayman, Brenda, R., N., Shudo, Jutaro, Howard, M., 2012. Age-Related Percutaneous Penetration Part 1: Skin Factor. Skin therapy letter. 17.

9. Lachman, L., Lieberman, H., A., dan Kanig, J., L., 1994. Teori dan Praktek Farmasi Industri, diterjemahkan oleh Siti Suyatmi edisi Ketiga, UI-Press, Jakarta.

10. Maraver, J., G., Cordero, M., D., Avila, M., O., Vega, A., F., Mala, M., Pavon, A., D., Miguel, M., D., CaleroC., P., Paz, M., V., Cotan, D., Alcazar, J., A.,S., 2014. Coenzyme Q10 Therapy. Molecular Syndromology. V 5.

11. Mehta, R., 2004. Topical and Transdermal Drug Delivery What a Pharmacist Needs to Know, http://www.inetCE.com, access 15 Agustus 2016.

12. M"uller, R.H., Radtke, M., Wissing, S.A., 2002 Nanostructured Lipid Matrices For Improved Microencapsulation Of Drugs. International Journal of Pharmaceutics. 242.

13. M"uller, R.H., Radtke, M., Wissing, S.A., 2002. Solid Lipid Nanoparticles (SLN) And Nanostructured Lipid Carriers (NLC) In Cosmetic And Dermatological Preparations. Advanced Drug Delivery. Rev. 54.

14. Patricia, S., Andreani, T., Sofia, A., M., Fanguerio, J., F., Halena, M., A., Santana,

2011.The current State of Art and New Trends on Lipid Nanoparticles (SLN and

NLC) for Oral Delivery. J of Pharm Res 7(8).

15. Patel, D., Dasgupta, S., Dey, S., Ramani, Y., R., Ray, S., Mazumder, B., 2012. Nanostructured Lipid Carrier(NLC)-Based Gel for the Topical Delivery of Aceclofenac: Preparation, Characterization, and In Vivo Evaluation. Scientia Pharmaceutica. 80.

16. Pezeshki, A., Ghanbarzadeh, A., Mohammadi, M., Fathollahi, I., Hamishehkar., H. 2014. Encapsulation of Vitamin A Palmitate in Nanostructured Lipid Carrier (NLC)-Effect of Surfactant Concentration on the Formulation Properties. Advanced Pharmaceutical Bulletin. 4 (Suppl 2)

17. Puglia, C., Blasi, P., Rizza, L., Schoubben, A., Bonina, F., Rossi, C., Ricci, M., 2008. Lipid nanoparticles for prolonged topical delivery: an in vitro and in vivo investigation. ScienceDirect. 375.

18. Shekhawat, P., B., 2013. Preparation And Evaluation of Clotrimazole Nanostructured Lipid Carrier for Topical Delivery. International Journal of Pharma and BioScience. 4 (1).

19. Shinde, G., Rajesh, K. S., Prajapati, N., Murthy, R., 2013. Formulation, Development, and 
Characterization of Nanostructured Lipid Carrier (NLC) Loaded Gel for Psoriasis. Der Pharmacia Lettre. 5 (4).

20. Sinko, Patrick. 2011. Physical Pharmacy and Pharmaceutical Science. 6th Ed. China: Lippincott Williams and Wilkins.

21. Talsania, Maulik Prabhubhai. 2013. Formulation And Optimization Of Nanostructured Lipid Matrices Of Repaglinide Using Factorial Design. Rajiv Gandhi University Of Health Sciences, Karnataka Bangalore. access www.rguhs.ac.in/cdc/...04 P052 34345.doc pada Agustus 2016.

22. Trommer, H., and Neubert, R.H.H., 2006. Overcoming The Stratum Corneum the Modulation of Skin Penetration. Skin Pharmacology and Physiology. 19.

23. Uprit S., Sahu R.K., Roy A., 2013. Preparation And Characterization Of Minoxidil Loaded Nanostructured Lipid Carrier Gel For Effective Treatment Of Alopecia. Saudi Pharmaceutical Journal. 21. 
Design of Nanostructured Lipid Carriers Ubiquinone-10 for Transdermal Treatment

\section{ORIGINALITY REPORT}

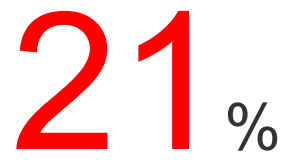

SIMILARITY INDEX

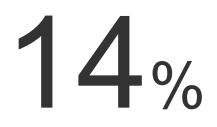

INTERNET SOURCES
$15 \%$

PUBLICATIONS
$0 \%$

STUDENT PAPERS

PRIMARY SOURCES

1

ajouronline.com

Internet Source

2 H. Trommer, R.H.H. Neubert. "Overcoming the

Stratum Corneum: The Modulation of Skin

$5 \%$

Penetration", Skin Pharmacology and

Physiology, 2006

Publication

3 www.inetce.com

Internet Source

4 dspace.nwu.ac.za

Internet Source

5 gnu.inflibnet.ac.in

Internet Source

6 es.scribd.com

Internet Source

Percutaneous Penetration Enhancers Chemical Methods in Penetration Enhancement, 2016. 
Hu, F.Q.. "Preparation and characteristics of monostearin nanostructured lipid carriers", International Journal of Pharmaceutics, 20060511

Publication

9

Nikhil Jain, Nisha Crouser, Elizabeth Yu.

"Lumbar Intervertebral Disc Herniation

Masquerading as an Epidural Hematoma",

JBJS Case Connector, 2018

Publication

10

Francesca Brugè, Elisabetta Damiani, Carmelo

Puglia, Alessia Offerta, Tatiana Armeni, Gian

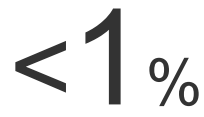

Paolo Littarru, Luca Tiano. "Nanostructured

lipid carriers loaded with CoQ10: Effect on

human dermal fibroblasts under normal and

UVA-mediated oxidative conditions",

International Journal of Pharmaceutics, 2013

Publication

11

Prasant Nahak, Gourab Karmakar, Priyam

Chettri, Biplab Roy et al. "Influence of Lipid

Core Material on Physicochemical

Characteristics of an Ursolic Acid-Loaded

Nanostructured Lipid Carrier: An Attempt To

Enhance Anticancer Activity", Langmuir, 2016

Publication

Amar-Yuli, Idit, Abraham Aserin, and Nissim

Garti. "Controlled Release and Delivery 
Technology of Biologically Active Proteins and Peptides", Bioactive Proteins and Peptides as

Functional Foods and Nutraceuticals

Mine/Bioactive Proteins and Peptides as

Functional Foods and Nutraceuticals, 2010.

Publication

13 www.dovepress.com

Internet Source

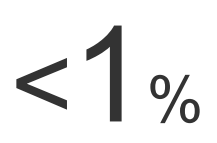

14 wjpr.net

Internet Source

$<1 \%$

15 Preeti Madhuri Pandey, Suraj Kumar Nayak,

Biswajeet Champaty, Indranil Banerjee, D. N.

Tibarewala, Kunal Pal. "chapter 12

Development of a Wireless Controlled

Iontophoretic Drug Delivery System", IGI

Global, 2018

Publication

16 Rohan Shah, Daniel Eldridge, Enzo Palombo, lan Harding. "Lipid Nanoparticles: Production,

Characterization and Stability", Springer Nature

America, Inc, 2015

Publication

17

refubium .fu-berlin.de

Internet Source

$<1 \%$

Shafaei, Zahra, Behafarid Ghalandari, Adeleh

Divsalar, and Ali Akbar Saboury. "Controlled 
release nutrition delivery based intelligent and targeted nanoparticle", Nutrient Delivery, 2017.

Publication

19

"Percutaneous Penetration Enhancers Drug

Penetration Into/Through the Skin", Springer

Nature, 2017

Publication

20 Isabel Almeida, Paulo Costa. "Tissue-based in

vitro and ex vivo models for dermal

permeability studies", Elsevier BV, 2016

Publication

21

C. Scott Asbill. "Transdermal Drug Delivery",

Pharmaceutical Manufacturing Handbook,

03/03/2008

Publication

22 ajprd.com

Internet Source

23 Jaiswal, Piyush, Bina Gidwani, and Amber Vyas. "Nanostructured lipid carriers and their current application in targeted drug delivery", Artificial Cells Nanomedicine and Biotechnology, 2015.

Publication 


\section{Design of Nanostructured Lipid Carriers Ubiquinone-10 for Transdermal Treatment}

GRADEMARK REPORT

FINAL GRADE

10

\section{PAGE 1}

PAGE 2

PAGE 3

PAGE 4

PAGE 5
GENERAL COMMENTS

Instructor 